\title{
A Mobility Management Scheme for Wireless Mesh Networks
}

\author{
Rongsheng Huang, Chi Zhang, and Yuguang Fang \\ Department of Electrical and Computer Engineering \\ University of Florida, Gainesville, FL 32611 \\ Tel: (352)392-8576; Fax: (352)392-0044 \\ rshuang@ufl.edu
}

\begin{abstract}
-
Current deployment of the wireless mesh networks (WMN) necessitates mobility management to support mobile clients roaming around the network without service interruption. Though Mobile IP and other previous protocols can be applied to WMNs to gain the micro-mobility as well as macro-mobility support, high signaling cost and long handoff latency problems still degrade the system performance significantly. In this paper we present a new mobility management scheme for WMNs, Mesh Mobility Management $\left(M^{3}\right)$. It utilizes some WMN's features and combines the per-host routing and tunneling techniques to reduce the signaling cost as well as to shorten the handoff latency. Our analysis shows that significant benefits can be achieved from this scheme.

Index Terms-Mobility Management, Micro-Mobility, Mobile IP, Wireless mesh networks.
\end{abstract}

\section{INTRODUCTION}

Over the past few years, wireless mesh networks (WMN) are gaining growing interest. This trend follows the popular needs for the inexpensive, continuous wireless wide-area coverage. A seamless wireless access is a common goal of the future communication.

Akyildiz et al. [3] have proposed a few models of WMNs. Usually, WMN consists of various types of entities: gateways, mesh routers, access points (AP) and mesh clients. Gateways are the connection points to the wire-line networks. Mesh clients are the terminal users which have no or limited routing function. Wireless APs are the entities in charge of the wireless access for the mesh clients. Stationary mesh routers form a wireless multihop backbone with long-range high-speed wireless techniques such as WiMAX. In different models, a mesh node can contain one or more functional entities, e.g., mesh routers usually implement AP functionalities.

When the mobile clients are stationary, with the support of backbone routing, the wireless access for them can be accomplished within a few hops. However, difficulty arises when there are needs for the mesh clients to move across the coverage area of different APs. How to maintain the ongoing connection and how to forward the downstream and upstream packets are not solved by the current standards. IEEE $802.16 \mathrm{e}$

This work was supported by the National Science Foundation under grant ANI-0093241 (Faculty Early Career Award) and under grant DBI-0529012. adds amendments to the original standard to support mobility, but only specifies MAC and PHY layer [1]. IEEE 802.11s attempts to extend the WiFi to support the mesh mode and provide mobility support, which is still under development.

Mobility management is not a new topic in other existing networks. Akyildiz et al. [2] presented a survey on this topic. In cellular systems, this part has already been a critical part to the continuous service of the mobile clients. Handoff quality is one of the most indispensable testing items in each field trial test. However, wireless mesh networks, which lack of infrastructure such as HLR and VLR, face more challenges in mobility management. Mobile IP is an approach which provides mobility support to mobile clients with IP identity [9]. The main idea is very similar to the HLR/VLR mechanism in cellular systems. Home Agent (HA) and Foreign Agent (FA) play the roles of home database and visiting database in the IP networks, respectively. Home address is used as the ID of a mobile client and the Care-of-Address (CoA) is used to locate the current position of the moving mobile clients.

Mobile IP can provide a solution to the inter-domain movement in WMNs. However, it is not suitable for the intra-domain movement, which is much more frequent than the inter-domain movement. The reason is that if FA is implemented in every AP, signaling cost and handoff latency become the major problems to the mobility support. Therefore, the solution to cope with the local movement is required. Protocols for IP micro-mobility have been proposed to solve the mobility dilemma in small-scale networks [6], [7], [10], [13]. Though these protocols can be applied to WMNs, heavier signaling cost and longer handoff latency due to more frequent local movement in WMNs still impede the practical mobility support.

In this paper, we propose a mobility management scheme in WMN, termed Mesh Mobility Management $\left(M^{3}\right)$. Some features of WMNs, such as multi-hop, mesh topology and continuous coverage, have been taken into consideration to better support the IP micro-mobility in WMNs.

The rest of this paper is organized as follows. Section II discusses some related works. Section III describes the proposed scheme. Performance analysis is carried out in Section IV. Conclusion is given at the end. 


\section{RElated WORKS}

In this paper, we focus on the mobility management within one WMN, which can be regarded as a micro-mobility issue. However, we search for a solution feasible with or without the Mobile IP support.

Not many related works of mobility management can be found in the literature of WMNs. Ganguly et al. [12] mentioned the mobility management issue in their comprehensive work. The experiment results confirm that handoff latency using a tunneling scheme is much longer than that using flat routing. However, since mobility management is not the focus of this paper, the authors discuss only the feasibility of mobility support and do not include detailed analysis. In SMesh [4], multiple APs monitor the moving mobile clients to achieve seamless handoff. This scheme eliminates the handoff latency at the price of high signaling cost.

However, previous works on IP micro-mobility are possible to be applied to WMNs, since WMN can be treated as one type of mobile IP networks. We now review some IP micromobility protocols.

In Cellular IP [13], mobile clients use the gateway's IP address as their CoA and each router in this domain use the home addresses of the mobile clients to route the downstream packets. The default routes for each router to the gateway are used to direct the upstream packets.

HAWAII is another important framework of IP micromobility [10]. The CoA of each mobile client in HAWAII is a unique IP address allocated by the gateway of the domain. Different from the Cellular IP, HAWAII uses the CoA of each mobile client to route the downstream packets. This difference makes HAWAII less coupled with Mobile IP protocol and also enables the per-flow QoS support in the backbone network.

In both schemes, each domain is identified by a single gateway and the entire domain is constructed to a tree-like structure. Both schemes require each router to maintain a routing entry for each mobile client in the downstream APs' coverage. When handoff occurs, the corresponding routing entries will be updated in all the routers involved from the new AP to the crossover router which is shared by the new AP and old AP. The invalid routing entries in the routers of the old path need to be removed. Due to the major feature of per-host routing, this type of schemes is called mobile-specific routing approach [5].

Another important type of IP micro-mobility protocols is the hierarchical tunneling approach [5], an example of which is Mobile IP Regional Registration (MIP-RR) [6]. Hsieh et al. [7] proposed another scheme, namely, Hierarchical Mobile IPv6. This type of schemes replaces the mobile-specific routing by introducing the tunneling technique. Through the hierarchical registration procedure, the higher-level FA knows the location information (ID of the lower-level FA) of the mobile clients and encapsulates the data packets with the destination address of this lower-level FA. Per-host routing entry is not required for the routers in these schemes while per-host location information is still stored in FAs. Due to the extra processing of encapsulation and decapsulation as in Mobile IP [9], larger delay is introduced to each flow. Additional cost of this type of schemes is that two or more CoAs have to be used. When handoff takes place, the registration with a different CoA also adds extra delay. The intuitive idea of this approach is to extend the Mobile IP mechanism to local movements.

\section{III. $M^{3}$ DESCRIPTION}

\section{A. Model Description}

In this paper we model the WMN with multiple mobile clients, one gateway, multiple routers with AP's functionality (called "AP" hereafter) and their covering area (called "cell" hereafter). The case of more than one gateway can be easily derived from this paper. Each AP has the functionality of AP, router and database for the subscriber information.

The gateway is required to assign a unique IP address in its domain to a mobile client when it is powered up. This unique IP address of a mobile client can be the CoA when mobile IP is provided for the inter-domain roaming. The foreign agent (FA) and home agent (HA) can reside in the gateway. In the scenario where more than one gateway present, our scheme can be easily extended by placing the FA/HA at the intersection of the gateways and using different IP address pools for each gateway.

We use a 3-level hierarchical structure to illustrate our scheme, as shown in Fig.1. The three APs connecting to the gateway have superior status than their downstream nodes. They are required to collect the location information of the mobile clients in the cells of the subordinate APs. We name these APs "superior routers (SR)" hereafter. The rest of the APs have equivalent status. SRs act as the delegates of the gateway and share the signaling traffic. In a smaller mesh network, if the gateway is not the bottle neck, these superior routers can be removed which yields only 2-level hierarchical structure.

As discussed in [11], a WMN can be constructed in a treelike structure. Each router has its only parent node and may have a number of children. This kind of modeling has its benefit for the routing where only the traffic flows between the gateway and each mobile client are considered. This model shows its limitation when the mobility management is taken into account. The tree structure is extracted from the real geographical topology based on the criterion of the shortest path from each AP to the gateway, which cannot be used to obtain the optimal path between any two geographical neighboring APs. The routing of previous schemes strictly follows the tree structure even when there exist shorter paths. Unlike other WMN models, our scheme allows the communication along the paths which are not in the tree. We assume that most of the time, geographically adjacent APs have shorter communication paths other than the only path along the tree. Therefore, this structure embodies a mesh topology.

We assume that the routing in the backbone (APs, superior routers and the gateway) has been set up. Since the backbone nodes in WMNs are mostly stationary, this assumption is reasonable. The remaining problem is on ensuring a mobile client to move around in this area without incurring high packet loss, long handoff latency and high signalling cost to the system. 


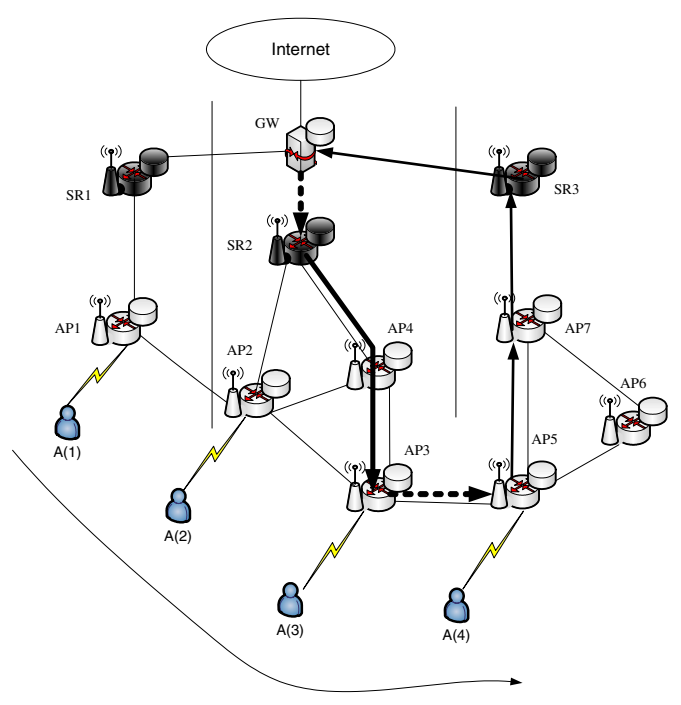

Fig. 1. Mesh Model and Illustration of $M^{3}$ Scheme

\section{B. Proposed Solution}

1) Power-up: We know that for a mobile client, the subscriber information should include authentication, authorization and accounting (AAA) information and QoS profiles. If every AP in the domain maintains a copy of all the mobile clients' subscriber information from the gateway, the network will be less scalable and difficult to administrate. In our scheme, when a mobile client is powered up the authentication procedure should be fulfilled before an IP address is allocated to this client according to the subscriber information in the gateway. The gateway activates the record of this mobile client and records the location information hereafter. The serving AP keeps a copy of the subscriber information to avoid frequent visiting the database in the gateway.

Database of each AP contains only the subscriber information of the mobile client currently in the cell. Database of each superior router additionally contains the location information of all the mobile clients residing in the subordinate APs' cells.

2) Handling Downstream Packets: The downstream packets, in which the destination address is not the AP's address, cannot be routed by the intermediate superior router and APs without routing entries. In this scheme, tunneling technique is used to forward the downstream packets. These packets are attached with extra IP headers in which the destination address is the destination AP's address. Upon receiving these tunneled packets, the destination APs decapsulate and forwards them to the addressed mobile clients in the cells.

In Fig. 1, the bold lines illustrate the downstream process, with the dashed lines and solid lines indicating the routing part and the tunneling part, respectively. From the gateway $(\mathrm{GW})$ to the SRs, the packets are routed according to the location information. The other routing part in downstream forwarding will be discussed in section 4).

3) Handling Upstream Packets: For the upstream packets, the tunneling is not needed. The APs can use the default routes to forward packets to the gateway.
4) Handling Handoff: Handoff occurs when the mobile client moves to a new AP's cell. Upon receiving a handoff request message from the moving client indicating the former AP's ID, the new AP sends a handoff request message to the former AP. The former AP sends back the corresponding subscriber information to the new AP after receiving the handoff request message. Meanwhile, it adds a temporary entry in its routing table with the destination address of this mobile client. A timer with length $T_{r}$ is started. After the timer expires, the routing entry and the corresponding subscriber information will be removed from the former AP. If the downstream packets are decapsulated by the former AP but the addressed mobile client is not found in the cell, these packets are routed to the new AP using the temporary routing entry. To guarantee that this routing can reach the new AP, each router on the path from the old AP to the new AP is required to add this routing entry. When the mobile client moves again, the chain of the downstream routes continues to be concatenated.The similar idea of this chain-like structure for the location update has been used by HMIP [8] and POFLA [14] for different applications. In cellular system, this method is called "pointer forwarding" and we borrow this name [14].

Suppose mobile client A moves from position 3 (A(3)) to position $4(\mathrm{~A}(4))$ in Fig. 1, the downstream packets are first tunneled to AP3. AP3 forwards the decapsulated packets to AP4 according to the "pointer" of mobile client A. Upstream packets from A are routed to gateway by the default routes of AP5, AP7 and SR3, sequentially.

To prevent the encapsulated packets from passing the final destination AP to a former AP, the attached IP header can include the mobile client's IP address in the option field. Therefore, when the encapsulated packets reach the final destination before the end of the tunnel, the AP of final destination can decapsulate them instead of simply passing them on.

5) Periodic Location Update: Adding the temporary routing entry is not the final solution to mobility management. Triangular routing problem is introduced by this method [9]. HMIP uses the idea that after a certain number of the hops the mobile client triggers a location update to the HA [8]. Our scheme uses the time interval to be the triggering condition. We assume that the mobile clients in the WMN are not so fast that during the period of $T_{l u}$ they cross less than $N_{h n d f}$ APs. Thereafter, once every $T_{l u}$, the mobile clients can trigger the location update to control the triangular routing problem. To make this update more efficient, we let each AP, instead of each mobile client, be the initiator to trigger this update. Each AP reports the current set of mobile clients to the superior routers. The superior routers select another interval $T_{h u}$ to periodically update the set to the gateway. $T_{h u}$ is obviously required to be no less than $T_{l u}$.

After this periodic location update, downstream packets can be tunneled to the AP where the mobile client exactly locates without traversing all the APs the mobile client has visited.

It is necessary to consider the case that if the time instant when each AP involved in a mobile client's handoff reports to its superior router is different, the downstream packets' routing might not follow the exact shortest route. The solution 
is simple. If $T_{r}$ is longer than the intervals of location update $T_{l u}$ and $T_{h u}$, the downstream packets can always find a path to the destination, which might not be the shortest. These extra hops can be expected less than $N_{h n d f}$.

\section{Extended Discussion of The Protocol}

Our scheme has combined the tunneling and per-host routing techniques which are the major features of previous two approaches [5].

Having compared these two types of approaches, we now discuss their pros and cons. By using the mobile-specific routing, the necessity of encapsulation/decapsulation is eliminated, and vice versa. The reason that mobile-specific routing cannot be applied to macro-mobility is the difficulty to find a crossover router which can maintain the mobile-specific routing entry. In other words, the scalability problem makes it infeasible. Moreover, this approach highly depends on the routing protocols. Another problem of this approach is pointed out in [5], which is, when update messages are lost due to physical reasons such as radio black-out, the routing entries in different routers might be inconsistent. Maintenance signaling might be an addition to guarantee the consistency. For the hierarchical tunneling approach, if the number of hierarchical levels are not small enough, the encapsulation/decapsulation will cause the delay performance intolerable. However, if the number of hierarchical levels are small enough, the signaling cost of handoff and handoff latency may be instead intolerable.

Our scheme achieves the advantages of both previous approaches. Tunneling the downstream packets in the backbone lower the routing requirement for each intermediate APs. Without the multiple-level registration procedure in the hierarchical tunneling approach, our scheme achieves shorter handoff latency. Consequently, the packet loss problem is greatly alleviated. A simple buffering technique can eliminate the packet loss without the out-of-order problem of packet forwarding. On the other hand, applying the per-host routing only between geographical neighboring APs does not require each AP to maintain too many intermediate routing entries. This "pointer forwarding" method significantly reduces the location update to the gateway despite the extra periodic location update which is introduced to control the triangular routing problem. The delay of downstream packets is controllable due to the controllable triangular routing.

The features of $M^{3}$ can be related to some features of WMNs. Continuous coverage is the reason for applying the mobile-specific routing in the last few hops. The stationary characteristic of WMNs' backbone yields simpler backbone routing. The hierarchical structure and the simple backbone routing render tunneling more appealing. Low speed of mobile clients limits the delay of downstream forwarding in this scheme. Moreover, due to the controllable delay, the periodic location update can be applied without side effects. Unlike the strict hierarchical structure of cellular system, mobility management under the physical mesh structure of WMNs can be realized in a more flexible way.

\section{Performance Analysis}

In what follows we focus on the benefits obtained related to the most important two factors in mobility management: signaling cost and handoff latency. We compare these two factors between our scheme and two previous approaches [5]. As we mentioned in the previous section, the encapsulation/decapsulation introduced by the tunneling brings us extra delay to downstream packets. This is the price when we want to use less per-host routing. However, this delay is not significant and should be tolerable because encapsulation and decapsulation only happen once for each downstream packet. Therefore, our performance analysis will not include this part.

The signaling cost is defined as the total amount of the extra signaling traffic due to the mobility. The handoff latency is defined as the service disruption time of the mobile clients due to handoff.

The signaling cost includes two major parts: update signaling cost during handoff and other maintenance signaling cost. The maintenance signaling in our scheme is the periodic location update signaling. To simplify the comparison, we calculate the signaling cost incurred during one period $T_{l u}$, with $T_{h u}=T_{l u}$ to further simplify the procedure.

In different mobility management schemes, the update procedure always starts from the new AP/BS to some anchor point, such as VLR/HLR or HA, then from the anchor point to the old AP/BS. If we assume the update signaling procedure is the same in different schemes, the cost depends only on the update path length, which is the focus our analysis.

Let $n, m, \rho, C, C_{u}, \overline{P L}$ denote the number of mobile clients, the number of APs, the handoff times per mobile client during one period $T_{l u}$, the total signaling cost, the update signaling cost during handoff, and the average signaling path length during handoff, respectively.

$$
\begin{gathered}
C=C_{u}+C_{\text {maintenance }} \\
C_{u}=\rho \cdot n \cdot \overline{P L}
\end{gathered}
$$

For our scheme, $\overline{P L_{M^{3}}}$ depends on the network planning and the geographical separation of neighbors. The worst case of this value occurs when all the available communication paths are those existing in the tree. However, usually in WMNs, the coverage is continuous and the geographical neighbors are interconnected to guarantee the connectivity so that each mobile client can reach the gateway in a limited number of hops. Therefore, $\overline{P L_{M^{3}}}$ can be assumed to be 1 .

Next,we procure $\overline{P L_{p s}}$ of the previous schemes and evaluate the gain by using our scheme under different traffic situation with different $\rho$.

In previous schemes, the communication always takes place along the tree paths. The average length from one node to its neighbors depends closely on the tree structure, such as the hierarchical level and the average number of children. Let $k$ denote the average number of children and $l+1$ the number of hierarchical levels as (including the level of gateway). We obtain the following equalities.

$$
m=\Sigma_{i=0}^{l} k^{i}=\frac{k^{l+1}-1}{k-1}
$$


Excluding the links in the tree, the number of which is $m-1$ (excluding the gateway), we define the handoff paths to be the sequential sibling visiting. The number of neighbor handoff paths with $2 \cdot j(j \in[1, l])$ hops can be expressed as follows.

$$
n_{2 j}=\Sigma_{i=1}^{l-j+1}(k-1) \cdot k^{j-1}=k^{l-j+1}-1
$$

Therefore, assuming that the handoff of each case is equal likely, the average path length can be expressed as:

$$
\overline{P L_{p s}}=\frac{2 \cdot \Sigma_{j=1}^{l} j \cdot\left(k^{l-j+1}-1\right)}{(m-1)-\frac{l \cdot(l+1)}{2}}
$$

The gain of our scheme is defined as $g=\frac{C_{p s}}{C_{M^{3}}}$. $C_{\text {maintenance }}$ in our scheme is the signaling cost of periodic location update, which is in the order of $\mathrm{m}$. Assume $\mathrm{n}$ is much larger than $\mathrm{m}$, with a factor of $a=n / m$. The gain $g$ will be approximately the ratio of $\frac{\overline{P L_{p s}}}{\overline{P L_{M}}}$ when $\rho$ becomes large enough. When $\rho$ is very small, the periodic update becomes the major signaling cost of handoff. The trend of $g$ under different $\rho$ can be shown in Fig.2.

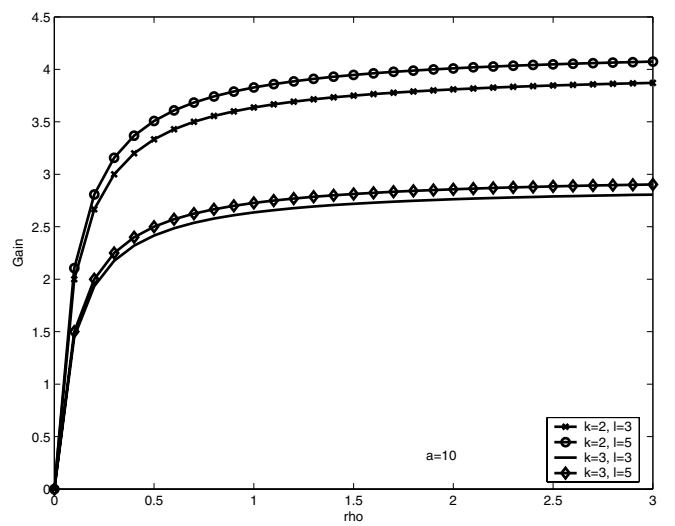

Fig. 2. Gain of $M^{3}$ over previous schemes

We now discuss the average path length. In the literature, the maximum number of hops is not recommended to be large, with 4 or 5 preferred. The average number of children $k$ should be relatively small in order to avoid the performance bottleneck. Table I shows the typical value set of the average path length.

TABLE I

Typical Value Set of Average Path Length

\begin{tabular}{|l|l|l|l|l|l|}
\hline parameters & $\begin{array}{l}\mathrm{k}=2, \\
\mathrm{l}=3\end{array}$ & $\begin{array}{l}\mathrm{k}=3, \\
\mathrm{l}=3\end{array}$ & $\begin{array}{l}\mathrm{k}=2, \\
\mathrm{l}=5\end{array}$ & $\begin{array}{l}\mathrm{k}=3, \\
\mathrm{l}=5\end{array}$ & $\begin{array}{l}\mathrm{k}=\mathrm{m}, \\
\mathrm{l}=1\end{array}$ \\
\hline $\begin{array}{l}\text { average } \\
\text { path } \\
\text { length }\end{array}$ & 4 & 2.9 & 4.21 & 3 & 2 \\
\hline
\end{tabular}

If there are normal routers (without AP functionality) in the network, the handoff between parents and children becomes less possible. Therefore, the average path length for the previous schemes will be larger. The gain of $M^{3}$ will be higher.
This signaling reduction is mainly for the SRs and the gateway of the WMN, which are the bottleneck most of the time.

Handoff latency can be expressed as:

$$
D=D_{h n d f-\operatorname{detc}}+D_{u-p a t h-u p d} \cdot \overline{P L^{\prime}}
$$

where $D_{h n d f-\operatorname{detc}}$ is the delay of handoff detection, $D_{u-p a t h-e s t b}$ is the unit-step delivery delay in path update and $\overline{P L^{\prime}}$ is the average length of path update.

Handoff detection is out of the scope of this paper. Therefore, we ignore the difference of $D_{h n d f-\operatorname{detc}}$ in different schemes. For the same reason as $\overline{P L_{M^{3}}}, \overline{P L_{M^{3}}^{\prime}}$ can be assumed to be 1 . For previous schemes, since the average position of the crossover routers is in the middle of the signaling path, we have $\overline{P L_{p s}^{\prime}}=0.5 \cdot \overline{P L_{p s}}$. Commonly, according to the typical value set of $\overline{P L_{p s}}$, our scheme performs better in handoff latency than previous schemes.

\section{Conclusion}

$M^{3}$ is proposed to meet the requirement of lower signaling cost and shorter handoff latency. This scheme utilizes some of the characteristics of the WMN to combine the two previous types of approaches. Consequently, it mitigates their shortcomings and achieves the advantages of both.

\section{REFERENCES}

[1] Ieee 802.16e: Air interface for fixed and mobile broadband wireless access systems. IEEE 802.16 Working Group, 2005.

[2] I. Akyildiz, J. McNair, J. Ho, H. Uzunalioglu, and W. Wang. Mobility management in next-generation wireless systems. Proceedings of the IEEE, 87(8):1347-1384, August 1999.

[3] I. Akyildiz, X. Wang, and W. Wang. Wireless mesh networks: a survey. Computer Networks, 47(4):445-487, March 2005.

[4] Y. Amir, C. Danilov, M. Hilsdale, R. Musaloiu-Elefteri, and N. Rivera. Performance of ip micro-mobility management schemes using host based routing. In Proceedings of the 4th international conference on Mobile systems, applications and services (MobiSys'06), Uppsala, Sweden, June 2006.

[5] A. T. Campbell, J. Gomez, S. Kim, C.-Y. Wan, Z. R. Turanyi, and A. G. Valko. Comparison of ip micro-mobility protocols. IEEE Wireless Communications, 9(1):72-83, February 2002.

[6] E. Gustafsson, A. Jonsson, and C. Perkins. Mobile ip regional registration. Internet Draft, 2000.

[7] R. Hsieh, A. Seneviratne, H. Soliman, and K. El-Malki. Performance analysis on hierarchical mobile ipv6 with fast-handoff over end-to-end tcp. In Globecom'02, Taipei, R.O.C., November 2002.

[8] W. Ma and Y. Fang. Dynamic hierarchical mobility management strategy for mobile ip networks. IEEE Journal on Selected Areas in Communications, 22(4):664-676, May 2004

[9] C. Perkins. Mobile ip. IEEE Communications Magazine, 35(5):84-99, May 1997.

[10] R. Ramjee, K. Varadhan, L. Salgarelli, S. R. Thuel, S.-Y. Wang, and T. L. Porta. Hawaii: a domain-based approach for supporting mobility in wide-area wireless networks. IEEE/ACM Transactions on Networking, 10(3):396-410, June 2002.

[11] A. Raniwala and T. Chiueh. Architecture and algorithms for an ieee 802.11-based multi-channel wireless mesh network. In Infocom'05, Miami, FL, March 2005.

[12] S.Ganguly, V. Navda, K. Kim, A. Kashyap, D. Niculescu, R. Izmailov, S. Hong, and S. Das. Performance optimizations for deploying voip services in mesh networks. IEEE Journal on Selected Areas in Communications, 24(11):2147-2158, November 2006.

[13] A. G. Valko. Cellular ip: a new approach to internet host mobility. Computer Communication, 29(1):50-65, Janurary 1999.

[14] W.Ma and Y. Fang. A pointer forwarding based local anchoring (pofla) scheme for wireless networks. IEEE Transactions on Vehicular Technology, 54(3):1135-1146, May 2005. 